REVIEW

\title{
New drugs for the treatment of epilepsy: a practical approach
}

\author{
S Beyenburg, J Baver, M Reuber
}

Postgrad Med J 2004;80:581-587. doi: 10.1136/pgmi.2004.019976

The availability of new antiepileptic drugs has broadened the spectrum of medical treatment options in epilepsy. The new agents, together with established drugs, offer substantial choice for doctors treating patients with focal or generalised epilepsy. The newer antiepileptic drugs are not necessarily more effective but usually better tolerated than the traditional agents, mainly because of favourable pharmacokinetic profiles and fewer interactions. Because treatment options have increased, drug therapy can now be tailored to the requirements of individual patients. Nevertheless, significant safety and efficacy issues continue to exist and there is a need for the development of even better agents. This review describes the clinical use of the new antiepileptic drugs, but focuses in particular on monotherapy, the treatment of generalised seizures, teratogenicity, and the cognitive side effect profile of the newer compounds.

See end of article for authors' affiliations ..........

Correspondence to: Dr S Beyenburg, Department of Neurology, Centre Hospitalier de Luxembourg, 4, rue Barblé, L-1210 Luxembourg; beyenburg. stefan@chl.lu

Submitted 3 February 2004 Accepted 4 March 2004
D espite recent developments in the nonmedical therapy of epilepsy, the prophylactic use of anticonvulsant drugs remains the mainstay of treatment. The licensing of several new antiepileptic drugs over the last two decades has not changed the basic treatment principles of epilepsy, but it has given patients and physicians greater choice. Focusing on issues of clinical relevance, it is the aim of this review to provide a comprehensive overview of the range of drug treatments now available.

The most important first step in the choice of an antiepileptic drug is the characterisation of seizure type(s) and epilepsy syndrome, as several antiepileptic drugs may have proconvulsive effects in the treatment of generalised seizures. ${ }^{1}$ The distinction of focal and secondary generalised seizures on the one hand and (idiopathic or primary) generalised seizures on the other is therefore of therapeutic relevance. Focal seizures are usually of symptomatic aetiology; primary generalised seizures predominantly occur in the context of idiopathic epilepsies (box 1). The determination of the epilepsy syndrome (for example, focal or idiopathic generalised epilepsy) does not only affect the choice of treatment but also allows a clearer prediction of the response to antiepileptic drugs, which is considerably better in idiopathic epilepsy. The new drugs have not changed these basic principles of epilepsy management, however, agents like lamotrigine, topiramate, levetiracetam, and felbamate have a broad spectrum of action with low proconvulsive potency.

The primary aim of treatment with antiepileptic drugs is the complete control of seizures over the long term. This aim is justified by the observation that quality of life improves with the achievement of seizure control. ${ }^{2}$ However, a comprehensive treatment of epilepsy cannot focus on this aim alone, as many other domains of life may be affected by a seizure disorder or its treatment-for instance, cognition, endocrine function, and mood. The aims of antiepileptic drug treatment are therefore more complex and better described as "complete freedom of seizures without negative effects on cognitive function, emotional, physical or general wellbeing" (box 2). In this respect, the new antiepileptic drugs have increased choice, allowing individual tailoring of treatment based on satisfactory anticonvulsant potency, but taking account of other health issues. As a result the modern medical therapy of epilepsy has become more complex.

Although based on fairly short term follow up and focusing on seizure control, some recent studies suggest that these aims can be achieved in the majority of patients. ${ }^{3}$ Monotherapy can control seizures fully in about $60 \%$ of patients with focal and secondary generalised seizures, or $75 \%$ to $95 \%$ of patients with generalised epilepsy. ${ }^{4}$ These figures reveal that, with an appropriately chosen antiepileptic drug, seizures in idiopathic generalised epilepsy are more easily treated, and that (at least over the short to medium term) medical treatment is also fairly successful in focal epilepsy. To date, no study has demonstrated that, in this respect, the new antiepileptic drugs have greater potency than more established anticonvulsant drugs. ${ }^{35}$

The treatment of the epilepsies becomes much more complex when an appropriately chosen first line drug given at an adequate or maximum tolerated dose has failed. Patients in this group are considered as having "difficult-to-treat" epilepsy, many will turn out to have "refractory" epilepsy over the longer term. One retrospective, observational study showed that only 13\% of patients who had failed to respond to initial monotherapy achieved seizure freedom with the first alternative monotherapy, and 3\% became seizure-free on combination therapy when serial monotherapy had failed. ${ }^{3}$ This result is in line

Abbreviations: GABA, gamma aminobutyric acid; JME, juvenile myoclonic epilepsy; NICE, National Institute for Clinical Excellence 
Box 1: Clinical features of focal and idiopathic generalised epilepsy

Focal (partial) epilepsy

- Onset at any age (most commonly in first year of life and $>65$ years).

- Evidence of other central nervous system pathology (injury, tumours, infection, dementia, etc).

- Aetiology mainly symptomatic or cryptogenic.

- Typical seizure types (examples):

- Focal seizures without impairment of consciousness.

- Focal seizures with impairment of consciousness.

- Secondary generalised tonic-clonic seizures (with focal onset).

\section{Generalised epilepsy}

- Onset most commonly in childhood, rarely $>30$ years.

- Not associated with other central nervous system pathology.

- Aetiology mainly idiopathic.

- Typical seizure types (examples):

- Absence seizures.

- Myoclonic seizures.

- Primary generalised tonic-clonic seizures.

with those of prelicensing randomised double blind add-on studies with the new anticonvulsants in which only $0 \%-8 \%$ of previously refractory patients became seizure-free (at least in the short term) whereas $20 \%-50 \%$ experienced a seizure reduction of $>50 \% .^{6}$ On the other hand, one smaller prospective study in which patients whose seizures continued on the first monotherapy were randomised to additional treatment with gabapentin or vigabatrin found that $31 \%$ and $39 \%$ of patients became seizure-free.

It seems that the tempting but confusing choice of combination therapy with two or more antiepileptic drugs only produces a very moderate gain in anticonvulsant potency in patients with refractory epilepsy who have already tried a number of drugs, although it is more likely to be effective in patients who continue to have seizures on their first monotherapy. To date no prospective studies have been conducted comparing alternative monotherapy and combination therapy.

It has been argued that antiepileptic drugs with different modes of action should be combined if polytherapy needs to be used (a concept known as "rational polytherapy"). ${ }^{8}$ However, current understanding of possible synergistic effects of different antiepileptic drugs is very limited. ${ }^{9-11}$ Moreover, it is difficult to translate pharmacological knowledge into clinical recommendations as the mechanism(s) of action of many of these drugs are not fully understood or completely unknown, and most exert their anticonvulsant effects in several different ways. It is easier to base therapeutic decisions on the lack of negative pharmacological interactions between the different drugs. In the end, both theoretical approaches lead to similar combinations, as the anticonvulsant properties of antiepileptic drugs which strongly induce hepatic enzymes (like carbamazepine and phenytoin) affect voltage gated sodium channels whereas the renally eliminated antiepileptic drugs-for instance gabapentin, vigabatrin, or levetiracetam-have different or unknown modes of action. Moderate enzyme inducers like lamotrigine, oxcarbazepine, tiagabine, and topiramate occupy
Box 2: Aims of treatment with antiepileptic drugs

- Complete freedom of seizures.

- Avoidance of negative effects on cognitive function, emotional, physical, or general wellbeing.

an intermediate position-as well as valproate, which has inhibitory effects on hepatic enzymes. The combination of carbamazepine with levetiracetam would therefore be a good choice for the treatment of focal seizures, as these drugs have different modes of action and side effect profiles, whereas the combination of carbamazepine and phenytoin would not be recommended. ${ }^{12}$

\section{MONOTHERAPY WITH NEW ANTIEPILEPTIC DRUGS}

In principle, anticonvulsant therapy in adults is usually considered after two unprovoked epileptic seizures. In view of the fact that the majority of patients can be treated successfully with one agent, ${ }^{3}$ there is a broad consensus of opinion that therapy should be started by choosing a single antiepileptic drug of first choice. The advantages of monotherapy are obvious-ease of concordance with a treatment plan, fewer interactions, and thereby fewer side effects. Moreover, monotherapy is less costly and has less teratogenic potential.

The choice of first agent is determined by seizure type, tolerability, efficacy, and patient characteristics like age, gender, contraception (see box 3), or family planning issues. This means that this choice is not exclusively based on anticonvulsant potency (which is similar for many drugs in this phase of treatment anyway) but also the other listed factors, including (increasingly) the cost of treatment. In the UK the monthly cost of treatment with carbamazepine or valproate is between $£ 2$ and $£ 15$ whereas the typical treatment costs with newer drugs are between $£ 50$ and $£ 100 .^{13}$ However, several studies suggest that lamotrigine, gabapentin, low dose topiramate, and possibly oxcarbazepine have superior tolerability.

The UK National Institute for Clinical Excellence (NICE), which assesses health technology used in the British National Health Service, recently considered how new antiepileptic drugs should be used based on the evidence available to date. ${ }^{13}$ The recommendations made are summarised in box 4 .

Of the new drugs, lamotrigine, oxcarbazepine, and topiramate are licensed as monotherapy in the treatment of epilepsy in the UK, gabapentin has a monotherapy licence in other countries. Monotherapy trials of other drugs-like levetiracetam-are currently under way. ${ }^{15}$ Vigabatrin is used as monotherapy for West's syndrome in children. Lamotrigine and topiramate as well as levetiracetam have a broad spectrum of activity and are not only used for focal but also for generalised seizures, for instance in the idiopathic epilepsies (several reviews have been published ${ }^{16-19}$ ). Felbamate is a further new drug with a broad spectrum of activity. However, it is not licensed for use in many countries because of problems with haematological and hepatic toxicity, although its efficacy in the monotherapy of refractory focal epilepsy was demonstrated in two conversion to monotherapy studies. ${ }^{20}$ Tiagabine, which increases synaptic and total brain gamma aminobutyric acid (GABA) by blocking GABA reuptake, ${ }^{21}$ failed to demonstrate its efficacy as monotherapy in a conversion-to-monotherapy study which followed on from a double blind randomised prelicensing trial. ${ }^{20}$ 
Box 3: Antiepileptic drugs and hormonal contraception ${ }^{14}$

Drugs that reduce the effectiveness of hormonal contraception

- Carbamazepine.

- Felbamate.

- Phenobarbital.

- Phenytoin.

- Primidone.

- Oxcarbazepine.

- Topiramate.

Drugs that do not reduce the effectiveness of hormonal contraception

- Ethosuximide.

- Gabapentin.

- Lamotrigine.

- Levetiracetam.

- Tiagabine.

- Valproate.

- Vigabatrin.

- Zonisamide.

- Benzodiazepines.

\section{EXPERIENCE WITH NEW ANTIEPILEPTIC DRUGS IN MONOTHERAPY \\ Gabapentin}

Gabapentin is a structural GABA analogue, which increases GABA synthesis and release and slows down GABA breakdown. ${ }^{21}$ It is usually well tolerated and effective in focal epilepsy, although it is considered to be of relatively low anticonvulsant potency. ${ }^{22}$ Clinical practice has shown that much higher doses than used in the premarketing studies can be effective. ${ }^{23}$ Several monotherapy studies have been completed (for a review see Gil-Nagel ${ }^{24}$ ). In patients with newly diagnosed epilepsy the drug is similarly effective as carbamazepine or lamotrigine. In one double blind study, the efficacy and tolerability of gabapentin (1200-3600 mg/day) was similar to that of lamotrigine $\left(100-300 \mathrm{mg} /\right.$ day). ${ }^{24}$ However, gabapentin did not prove its efficacy in monotherapy in a conversion-to-monotherapy study following on from a prelicensing double blind randomised controlled study in patients with refractory epilepsy. ${ }^{20}$

\section{Assessment}

Gabapentin is effective as add-on treatment or in the monotherapy of newly diagnosed focal epilepsy. It is not indicated in generalised epilepsy syndromes (especially absence epilepsies). It has favourable pharmacokinetic properties (lack of drug interactions, no hepatic metabolism) and good tolerability allowing rapid dose escalation. In view of its pharmacokinetic features and the fact that it is not metabolised in the liver, its use may especially be considered in multimorbid patients treated with other pharmacological agents and in patients with liver disorders. Its good tolerability may also be particularly important in older people with epilepsy. In this group of patients, the tolerability may be improved further by increasing the daily dose very slowly.

\section{Lamotrigine}

Like phenytoin or carbamazepine, lamotrigine exerts most of its antiepileptic activity by blocking excitatory $\mathrm{Na}^{+}$channels in a voltage and use dependent manner. It also reduces $\mathrm{Ca}^{2+}$
Box 4: UK NICE recommendations for the use of new antiepileptic drugs published in $2002^{13}$ :

New antiepileptic drugs should be considered within their licensed indications:

- If established drugs (typically carbamazepine or valproate) have failed.

- If the most appropriate older drug is contraindicated.

- If an older drug could interact with other medications (including the oral contraceptive pill).

- If the older drugs are already known to be poorly tolerated by the individual.

- If the patient is a woman of childbearing potential (although the safety of newer antiepileptic drugs in pregnancy remains unclear).

currents and may have additional unrecognised effects, which may explain its broad spectrum of effectiveness. ${ }^{21}$ Several randomised monotherapy studies have compared lamotrigine with carbamazepine or phenytoin. ${ }^{18}{ }^{24} 26-30$ These studies showed that, while lamotrigine is similarly effective in the treatment of focal epilepsy as carbamazepine or phenytoin, it is better tolerated-a fact reflected in lower rates of treatment discontinuation in lamotrigine treated patients. ${ }^{24} 31$ Lamotrigine also has a favourable side effect profile when compared with valproate, as it does not cause weight gain and has no or fewer adverse effects on sex hormones in women, is likely to have a lower teratogenic potential, and causes less cognitive dysfunction. ${ }^{32-37}$ Although (at least in the treatment of idiopathic generalised epilepsy) some clinicians consider lamotrigine to have lower anticonvulsant potency than valproate, there are at present no randomised head-to-head studies comparing the effectiveness of the two drugs. A direct comparison of lamotrigine in doses ranging from 100 to $300 \mathrm{mg} /$ day and gabapentin (1200 to $3600 \mathrm{mg} /$ day) showed no difference between the two drugs in terms of tolerability and effectiveness in patients. ${ }^{25}$

\section{Assessment}

After over 10 years of clinical experience, lamotrigine has demonstrated its usefulness as a well tolerated antiepileptic drug (with the exception of the risk of allergic-toxic reactions during the initiation period). ${ }^{18} 24$ Further strengths of lamotrigine are its positive psychotropic effects, ${ }^{18}{ }^{38-40}$ its broad spectrum of action, and its favourable side effect profile, especially in women and older people. Lamotrigine may be preferable to valproate in puberty in view of the lack of endocrine effects. It is a disadvantage of treatment with lamotrigine that the dose has to be built up slowly to minimise the risk of allergic-toxic reactions and that patients are therefore not protected effectively against seizures for the first two months of treatment. Furthermore, plasma concentrations of lamotrigine are reduced significantly by a coadministered oral contraceptive pill. ${ }^{41}$

\section{Oxcarbazepine}

Oxcarbazepine is thought to exert its antiepileptic activity through blockage of voltage sensitive sodium channels. It also reduces presynaptic glutamate release. ${ }^{21}$ Numerous studies have demonstrated the effectiveness of oxcarbazepine in the monotherapy of focal epilepsy in children and adults (see recent reviews ${ }^{19}{ }^{42} 43$ ). Oxcarbazepine represents a structural modification of the carbamazepine molecule and has similar potency but a more favourable side effect profile (with the exception of hyponatraemia). Patients can be switched directly over from carbamazepine to oxcarbazepine 
(using a ratio of 1:1.5 carbamazepine: oxcarbazepine up to $1500 \mathrm{mg} /$ day carbamazepine and of 1:1 for higher doses), although some clinicians advocate a more gradual switch in patients taking more than $800 \mathrm{mg} /$ day of carbamazepine or patients taking more than one antiepileptic drug. ${ }^{42}{ }^{44}$ Patients switched from carbamazepine to oxcarbazepine may experience improvements in terms of tolerability or effectiveness. ${ }^{45}$ The potential for drug-drug interactions is lower with oxcarbazepine than carbamazepine. Fewer patients treated with oxcarbazepine than carbamazepine develop allergic reactions, however, the rate of cross allergy is $25 \%$. Oxcarbazepine causes hyponatraemia in up to $2.7 \%$ of patients. Although this is not clinically relevant in the majority of cases, oxcarbazepine associated hyponatraemic coma has been reported and hyponatraemia may be more problematic in older, multimorbid patients or those with cardiac or renal disease in whom regular blood electrolyte estimations are indicated. ${ }^{42}{ }^{46}{ }^{47}$ Head-to-head monotherapy studies (oxcarbazepine $v$ valproate, oxcarbazepine $v$ carbamazepine, oxcarbazepine $v$ phenytoin) in patients with newly diagnosed epilepsy showed that oxcarbazepine was better tolerated than carbamazepine and phenytoin and similarly well tolerated as valproate. There were no clear differences between the drugs in terms of effectiveness (for a review see Kwan and Brodie $^{19}$ ). Oxcarbazepine has weaker enzyme inducing effects than carbamazepine and therefore affects sex hormone metabolism less significantly than carbamazepine. ${ }^{48}$ In men with epilepsy especially, it has been shown that testosterone levels, which are reduced in temporal lobe epilepsy, are lowered further by carbamazepine. ${ }^{49}$ This could have relevant reproductive effects. It is possible that such negative effects could be avoided in patients treated with oxcarbazepine.

\section{Assessment}

Oxcarbazepine is a potent and well tolerated drug for the treatment of focal epilepsy. In monotherapy, doses can be escalated relatively swiftly, although particular care has to be taken in older, multimorbid patients. Oxcarbazepine is not indicated in idiopathic generalised epilepsy syndromes (especially in absence seizures), which it may exacerbate. ${ }^{42}$ In general, oxcarbazepine is better tolerated than carbamazepine and has less potential for drug interactions. Blood sodium concentrations should monitored in patients with known hyponatraemia, elderly and multimorbid patients, patients taking other drugs with effects on sodium levels, and those with possible symptoms of hyponatraemia.

\section{Topiramate}

Topiramate exerts its antiepileptic effects by influencing the activity of some types of voltage gated $\mathrm{Na}^{+}$and $\mathrm{Ca}^{2+}$ channels, $\mathrm{GABA}_{\mathrm{A}}$ receptors, and certain glutamate receptors. It also inhibits some isoenzymes of carbonic anhydrase. ${ }^{50}$ Topiramate is licensed for use in the monotherapy of focal epilepsy in adults in the UK and several other countries. Topiramate is much better tolerated when used on its own than in combination therapy, which is often limited by the negative cognitive effects of this highly potent anticonvulsant. ${ }^{39}$ In general, monotherapy studies show that topiramate in daily doses of 100 to $200 \mathrm{mg}$ /day is as effective as relatively low doses of carbamazepine $(600 \mathrm{mg} /$ day $)$ and valproate ( $1250 \mathrm{mg} /$ day) in children and adults, irrespective of seizure type..$^{51-54}$ So far, no studies have revealed that topiramate monotherapy is superior to standard antiepileptic drugs in monotherapy. ${ }^{55}$

\section{Assessment}

Topiramate is a potent antiepileptic drugs with a broad spectrum of activity. In monotherapy, at least in doses up to $100 \mathrm{mg} /$ day, it is better tolerated than carbamazepine and valproate. More studies of tolerability in higher doses are required to ensure that the negative cognitive effects seen in combination therapy do not also affect patients treated with monotherapy. Paraesthesias (relatively common in monotherapy) and weight loss are side effects specific to topiramate. Patients should also be informed about an increased risk of renal stones caused by carboanhydrase inhibition. ${ }^{55}$ Doses of less than $200 \mathrm{mg} /$ day do not interfere with the effectiveness of the oral contraceptive pill. If higher doses of topiramate are used in monotherapy, the oestrogen content of the oral contraceptive pill should be above $50 \mu \mathrm{g}$ and patients should be warned about the significance of breakthrough bleeding (which indicates that contraception is not effective).

\section{Levetiracetam}

The mechanism of action of levetiracetam is poorly understood. There is also insufficient information about levetiracetam monotherapy from valid clinical studies. However, the clinical experience with levetiracetam so far suggests that it is a well tolerated and highly effective drug for the treatment of focal, and probably also of generalised epilepsy. ${ }^{15} 1656$ To date, there are no head-to-head studies of levetiracetam with standard or other new antiepileptic drugs in monotherapy.

\section{Assessment}

At present there are insufficient data about levetiracetam monotherapy. However, in view of its almost ideal pharmacokinetic properties (no known interactions, no hepatic metabolisation), its good tolerability and its very broad spectrum of activity, levetiracetam promises to be a very useful drug for the monotherapy of many different seizure types and epilepsy syndromes. ${ }^{15} 16$

\section{Vigabatrin}

Vigabatrin increases GABA levels by binding irreversibly to GABA transaminase.$^{20}$ Of all new drugs vigabatrin is the only one which has shown superior potency to conventional drugs. However, this evidence is limited to its use in West's syndrome. ${ }^{57}$ It is now the monotherapy agent of first choice for this indication. Otherwise, vigabatrin is only used as a drug of last choice in patients with refractory focal epilepsy (that is, when other appropriate monotherapies and combination treatments have failed). The reason for this is that vigabatrin causes irreversible visual field defects in 30\% to $60 \%$ of patients, which are thought to be due to GABA mediated toxic effects on retinal amacrin cells. ${ }^{58} 59$

\section{FUTURE PERSPECTIVES}

The evidence base for the most effective use of some of the new antiepileptic drugs (lamotrigine, gabapentin, topiramate, and oxcarbazepine) in newly treated patients with epilepsy will improve significantly when the results of the study of Standard And New Antiepileptic Drugs (SANAD, www.liv.ac.uk/neuroscience/sanad) are analysed in 2005. This randomised controlled trial compares the longer term clinical outcomes and cost effectiveness of treatment with carbamazepine or valproate with that of new drugs in around 3000 patients. Two further antiepileptic drugs will become available in some European countries and the UK in the near future, zonisamide and pregabalin. It is likely that they will initially be licensed as adjunctive treatments in focal epilepsy, although zonisamide has also been reported to be effective in generalised epilepsies. It will take some time before their role in the monotherapy of epilepsy will become clear. Zonisamide principally seems to exert its antiepileptic effects by blocking voltage gated $\mathrm{Na}^{+}$and $\mathrm{Ca}^{2+}$ channels. ${ }^{60}$ The antiepileptic effects of pregabalin may depend on a reduction of cellular glutamate. ${ }^{61}$ Although a number of other substances are 
currently under preclinical evaluation, ${ }^{12}$ it is likely that zonisamide and pregabalin will be the last new antiepileptic drug to enter clinical use for some time.

\section{ROLE OF NEW ANTIEPILEPTIC DRUGS IN THE TREATMENT OF PRIMARY (IDIOPATHIC) GENERALISED EPILEPSIES}

There is scant evidence about the use of new antiepileptic drugs for primary (idiopathic) generalised epilepsies from prospective studies. Clinical experience, open label studies, and a small number of controlled studies suggest that lamotrigine, topiramate, felbamate, and probably also levetiracetam, ${ }^{1756}$ may be effective, at least for primary generalised tonic-clonic seizures. Lamotrigine and topiramate are now licensed for this indication. The effectiveness of these drugs in the treatment of absence epilepsies or juvenile myoclonic epilepsy (JME) is a matter of controversy. Valproate seems superior in terms of anticonvulsant potency. ${ }^{17}$ The use of lamotrigine in the treatment of myoclonic seizures is particularly problematic. ${ }^{62}$ However, if patients fail to tolerate valproate or there are other relative contraindications to valproate (for example, reproductive disorders, weight gain) these new antiepileptic drugs may be considered. The evidence for clinical effectiveness in generalised epilepsies is currently strongest for lamotrigine (see published reviews ${ }^{17-19}$ ). However, it has been observed that lamotrigine may cause an increase in myoclonic seizures in JME (Janz syndrome). ${ }^{6364}$

Although topiramate has been shown to have a broad spectrum of anticonvulsant activity in animal experiments, ${ }^{65}$ there is insufficient experience with its clinical application in the treatment of primary generalised epilepsies at present, although it has been licensed for this indication in some countries. Smaller, open label studies, anecdotal reports, and one placebo controlled study suggest that topiramate has beneficial effects in generalised epilepsies. ${ }^{66} 67$ One placebo controlled study described the effects of this in 80 patients. Fifty six percent of the topiramate treated patients had experienced a greater than $50 \%$ seizure reduction after 16 weeks (as opposed to $20 \%$ in the placebo group) ${ }^{67}$ In children topiramate can be effective in various generalised epilepsy syndromes when other anticonvulsants have failed. ${ }^{68}$

Levetiracetam also has a broad spectrum of action in animal experiments, so that-although not currently licensed for this indication-its use in generalised epilepsy could be considered. ${ }^{16}{ }^{56}$ Anecdotal reports and case series suggest that levetiracetam is effective in primary generalised epilepsies. $^{56}{ }^{6970}$ Doses of $1000 \mathrm{mg} /$ day to $4000 \mathrm{mg}$ /day were used in previously refractory patients. So far, there are no studies describing longer term (greater than one year) treatment results in primary generalised epilepsy with levetiracetam. Many other reports about the use of levetiracetam in this setting have only been published in abstract form (for reviews see Ben-Menachem and Bourgeois ${ }^{16}{ }^{17}$ ). Despite the obvious methodological deficiencies of the studies analysing levetiracetam therapy in primary generalised epilepsy to date (February 2004), the use of this drug may be considered as co-medication in otherwise refractory patients with generalised epilepsies, especially as many of the few alternative agents have a more negative side effect profile.

\section{EFFECTS ON COGNITIVE FUNCTIONS AND BEHAVIOUR}

Apart from their "cleaner" pharmacokinetic profile, the most important reason why the new antiepileptic drugs may be preferable to the more established drugs is their favourable side effect profile. The most relevant side effects are central nervous system related and affect cognitive functions. Unfortunately, there are only a small number of prospective studies investigating the effects of new drugs on cognition and behaviour. One recent review pointed out that, in the absence of head-to-head studies, methodological flaws and differences between different existing studies made a comparison of the effects of "new" and "old" antiepileptic drugs very difficult. ${ }^{71}$ With the exception of topiramate, there appears to be a tendency for newer drugs to have less negative effects on cognitive functions than conventional drugs. The effects of tiagabine, gabapentin, and especially levetiracetam have not been examined sufficiently to form a preliminary, even less a firm view of their potential to interfere with cognitive functions. ${ }^{39} 7172$ There are considerably more data on lamotrigine, topiramate, and (although to a lesser extent) on oxcarbazepine. The latter drug appears to have no relevant negative cognitive effects on healthy volunteers or on patients with newly diagnosed epilepsy. ${ }^{39} 71$ Although the frequency and intensity of topiramate induced cognitive problems and psychiatric disorders is in part dependent on the rate of dose escalation (the risk of cognitive dysfunction with predominant word finding difficulties can be reduced if the dose is built up by no more than $25 \mathrm{mg}$ per week or fortnight), it is clear that the drug has a considerable potential to cause cognitive side effects and changes in behaviour. ${ }^{39}{ }^{71-74}$ Apart from the speed of dose escalation, a family history of psychiatric disorder and epilepsy, a history of febrile convulsions and generalised tonic-clonic seizures have been shown to be risk factors for the development of topiramate related cognitive problems. ${ }^{74}$ In contrast, lamotrigine has the most favourable profile of cognitive effects including positive effects on different aspects of cognition and antidepressant effects. ${ }^{34} 38-407175$

\section{SPECIFIC SIDE EFFECTS}

While the listed studies and clinical experience with the new drugs show that they are well tolerated by more patients than the established drugs, most new drugs can be associated with idiosyncratic side effects (table 1). The recently described topiramate associated acute ocular syndrome with reduced visual acuity, myopia, and increased intraocular pressure is very rare and reversible if the drug is discontinued. ${ }^{76}$ The cause of this condition is unknown. Several reports suggest that topiramate given in combination with valproate, and possibly also with other drugs metabolised in the liver, can be hepatotoxic. ${ }^{77}$ The visual field problems associated with vigabatrin have been discussed above. ${ }^{58} 59$ Although levetiracetam was remarkably well tolerated in prelicensing studies and caused few psychiatric side effects, ${ }^{78}$ its use in clinical practice has uncovered potential problems: apart from reports about allergic-toxic reactions, ${ }^{79}$ and seizure exacerbation, ${ }^{80}$ psychopathological side effects like irritability, aggressiveness, and even (reversible) psychosis are sometimes seen (especially in patients with a history of psychiatric disorders). ${ }^{81-83}$ Finally some patients complain of somnolence and sedation which may occur and persist even at low doses of levetiracetam.

\section{TERATOGENICITY}

There are insufficient data to make definitive statements about the safety of new antiepileptic drugs in pregnancy. In view of this lack of information, caution is advised. A detailed, second trimester fetal anomaly ultrasound scan is often recommended but may give a false sense of certainty and can confront people treated with anticonvulsants with a difficult dilemma if an abnormality is detected. International, US, and UK pregnancy registers are in the process of collecting data which should allow better informed pregnancy counselling in the future. In the North American monotherapy register, lamotrigine was associated with a $1.8 \%$ risk of abnormalities (compared with $8.6 \%$ in babies 
Table 1 Idiosyncratic reactions and important long term side effects of the new antiepileptic drugs

\begin{tabular}{|c|c|}
\hline Vigabatrin & $\begin{array}{l}\text { Irreversible concentric visual field deficits, depression, } \\
\text { psychosis, weight gain, tremor }\end{array}$ \\
\hline Lamotrigine & $\begin{array}{l}\text { Allergic-toxic reactions, headache, tremor, vomiting, } \\
\text { insomnia }\end{array}$ \\
\hline Gabapentin & Weight gain, behavioural abnormalities (infrequent) \\
\hline Felbc & $\begin{array}{l}\text { Aplastic anaemia, hepatotoxicity, anorexia, } \\
\text { gastrointestinal symptoms }\end{array}$ \\
\hline Tiago & Status epilepticus, fatigue \\
\hline Topiramate & $\begin{array}{l}\text { Cognitive disturbance, weight loss, renal stones, } \\
\text { paraesthesia, ocular syndrome (myopia and increased } \\
\text { intraocular pressure) }\end{array}$ \\
\hline Oxcarbazepine & Allergy, hyponatraemia \\
\hline Levetiracetam & $\begin{array}{l}\text { Behavioural abnormalities, psychosis, tremor, allergy } \\
\text { (all infrequent), sedation }\end{array}$ \\
\hline Zonisamide & Fatigue, paraesthesiae, renal stones \\
\hline
\end{tabular}

exposed to valproate and $12 \%$ to those with phenobarbital exposure). ${ }^{36}$ The rate of malformations in lamotrigine monotherapy exposed pregnancies registered in the international register was 3\% (31 March 2003). ${ }^{36}$ This rate was identical in the prospective British register $(95 \%$ confidence interval $1.5 \%$ to $5.7 \%$ ) compared with $7.2 \%$ in pregnancies exposed to valproate $(95 \%$ confidence interval $5.2 \%$ to $10.0 \%) .{ }^{36}$ The combination of lamotrigine and valproate was associated with a malformation risk of $11.9 \%$ or $10.4 \%$ respectively, ${ }^{36}$ the combination of lamotrigine with other antiepileptic drugs with a risk of $3.4 \% .{ }^{36}$ Despite these data, lamotrigine should only be used in pregnancy after a careful assessment of the potential risks and benefits.

\section{CONCLUSION}

The development of new antiepileptic drugs has not changed the basic principles of the medical therapy of epilepsy (box $5)$, but it has substantially increased treatment choice. So far, it does not appear that the new drugs have greater anticonvulsant potency than conventional agents. However, the new drugs have a more favourable side effect profile, which may represent a significant advantage in the treatment

\section{Box 5: Basic principles of the medical treatment of epilepsy}

- Determine the need for antiepileptic treatment (consider antiepileptic drugs after more than one unprovoked seizure, a single seizure in the presence of identifiable intracranial lesion, presentation with status epilepticus).

- Determine seizure type and epilepsy syndrome (focal/ generalised/unclassifiable) and chose appropriate first line antiepileptic drug (broad spectrum in unclassifiable epilepsies).

- Increase antiepileptic drug to an appropriate dose.

- If ineffectual, consider further treatment options: 1. Increase to fully effective/maximum tolerated dose. 2. Switch to alternative monotherapy. 3. Chose appropriate add-on antiepileptic drugs.

- If ineffectual, consider non-medical treatment options (especially epilepsy surgery for refractory focal epilepsies).

- If combination antiepileptic drugs therapy fails to achieve full seizure control, consider reverting to most effective monotherapy. of a chronic disorder. It remains to be demonstrated that this potential advantage outweighs the considerably greater costs of "modern" antiepileptic therapy. Moreover, longer term side effects may only become apparent with continuing use of these new drugs (the vigabatrin associated irreversible visual field defects are an example of this). The choice of treatments now available enables physicians to take account of their patients' particular wishes and circumstances. Most of the new agents have better pharmacokinetic properties than conventional antiepileptic drugs, including fewer interactions with other drugs. This facilitates combination therapy when two or more drugs are necessary. In particular lamotrigine, gabapentin, and levetiracetam do not interfere with the effectiveness of the oral contraceptive pill, topiramate, and oxcarbazepine have less potent effects on contraceptive hormone levels than carbamazepine and phenytoin. On the other hand, knowledge on the safety of the new antiepileptic drugs in pregnancy remains deficient (with the possible exception of lamotrigine), so that patients can not really be informed about the risk of malformations associated with most of the new drugs. Some of the new antiepileptic drugs (especially lamotrigine and gabapentin) have fewer negative cognitive effects than standard anticonvulsants. The dose of several of the new drugs can be increased relatively quickly, at least if they are used in monotherapy, providing rapid anticonvulsant protection (gabapentin, oxcarbazepine), or their broad spectrum of action allows them to be used in focal as well as idiopathic generalised epilepsies (lamotrigine, topiramate, levetiracetam). There is an urgent need for appropriately powered prospective studies to guide the clinical use of the new antiepileptic drugs, and to enable physicians to chose between conventional and new drugs. ${ }^{22}$

\section{Authors' affiliations}

S Beyenburg, Department of Neurology, Centre Hospitalier de Luxembourg, Luxembourg

J Bauer, Department of Epileptology, University of Bonn, Germany M Reuber, Department of Neurology, Sheffield Teaching Hospitals NHS Trust, Royal Hallamshire Hospital, Sheffield, UK

\section{REFERENCES}

1 Baver J. Seizure-inducing effects of antiepileptic drugs: a review. Acta Neurol Scand 1996;94:367-77.

2 Gilliam FG. The impact of epilepsy on subjective health status. Curr Neurol Neurosci Rep 2003;3:357-62.

3 Kwan P, Brodie MJ. Early identification of refractory epilepsy. N Engl J Med 2000;342:314-19.

4 Baver J. Antiepileptische Kombinationstherapie. Rationale Konzepte versus reelle Effektivität. Fortschr Neurol Psychiatr 1998;66:414-26.

5 Schmidt D. The clinical impact of new antiepileptic drugs after a decade of use in epilepsy. Epilepsy Res 2002;50:21-32.

6 Marson AG, Kadir ZA, Hutton JL, et al. The new antiepileptic drugs: a systematic review of their efficacy and tolerability. Epilepsia 1997:38:859-80.

7 Lindberger M, Alenius M, Frisen L, et al. Gabapentin versus vigabatrin as first add-on for patients with partial seizures that failed to respond to monotherapy: a randomised, double-blind, dose titration study. GREAT Study Investigators Group. Gabapentin in refractory epilepsy add-on treatment. Epilepsia 2000;41:1289-95

8 Stephen $L$, Brodie M. Seizure-freedom with more than one antiepileptic drug. Seizure 2002;6:349-51.

9 Brodie MJ, Yuen AW. Lamotrigine substitution study: evidence for synergism with sodium valproate? 105 study group. Epilepsy Res 1997;26:423-32.

10 Czuczwar SJ, Borowicz KK. Polytherapy in epilepsy: the experimental evidence. Epilepsy Res 2002;52:15-23.

11 Gupta AK, Jeavons PM. Complex partial seizures: EEG foci and response to carbamazepine and sodium valproate. J Neurol Neurosurg Psychiatry 1985;48:1010-14.

12 Bauer J, Reuber M. Medical treatment of epilepsy. Expert Opin Emerg Drugs 2003:8:457-67.

13 National Institute of Clinical Excellence. Final appraisal determinationnewer drugs for epilepsy in adults. http://www.nice.org.uk/pdf/ Epilepsy_adult_FAD.pdf.

14 Crawford P. Interactions between antiepileptic drugs and hormonal contraception. CNS Drugs 2002;16:263-72.

15 Ben-Menachem E. Preliminary efficacy of levetiracetam in monotherapy. Epileptic Disord 2003;5(suppl 1):S51-S55. 
16 Ben-Menachem E. Levetiracetam: treatment in epilepsy. Expert Opin Pharmacother 2003;4:2079-88.

17 Bourgeois BF. Chronic management of seizures in the syndromes of idiopathic generalized epilepsy. Epilepsia 2003:44(suppl 2):S27-32.

18 Choi H, Morrell MJ. Review of lamotrigine and its clinical applications in epilepsy. Expert Opin Pharmacother 2003;4:243-51.

19 Kwan P, Brodie MJ. Clinical trials of antiepileptic medications in newly diagnosed patients with epilepsy. Neurology 2003;60(suppl 4):S2-S12.

20 Beydoun A, Kutluay E. Conversion to monotherapy: clinical trials in patients with refractory epilepsy. Neurology 2003;60:S13-25.

21 Kwan P, Sills GJ, Brodie MJ. The mechanisms of action of commonly used antiepileptic drugs. Pharmacol Ther $2001 ; 90: 21-34$.

22 Marson AG, Chadwick DW. New drug treatments for epilepsy. J Neurol Neurosurg Psychiatry $2001 ; 70: 143-48$.

23 Wilson EA, Sills GJ, Forrest $G$, et al. High dose gabapentin in refractory partial epilepsy: clinical observations in 50 patients. Epilepsy Res 1998;29:161-66.

24 Gil-Nagel A. Review of new antiepileptic drugs as initial therapy. Epilepsia 2003;44(suppl 4):3-10.

25 Brodie MJ, Chadwick DW, Anhut H, et al. for the Gabapentin Study Group 945-212. Gabapentin versus lamotrigine monotherapy: a double-blind comparison in newly diagnosed epilepsy, Epilepsia 2002;43:993-1000.

26 Brodie MJ, Richens A, Yuen AW. Double-blind comparison of lamotrigine and carbamazepine in newly diagnosed epilepsy. UK Lamotrigine/ Carbamazepine Monotherapy Trial Group. Lancet 1995:345:476-79.

27 Brodie MJ. A multicenter double-blind randomized comparison between lamotrigine and carbamazepine in elderly patients with newly diagnosed epilepsy. Epilepsia 1998;39(suppl 6):72.

28 Reunanen M, Dam M, Yuen AW. A randomised open multicentre comparative trial of lamotrigine and carbamazepine as monotherapy in patients with newly diagnosed or recurrent epilepsy. Epilepsy Res 1996;23:149-55.

29 Steiner TJ, Dellaportas $\mathrm{Cl}$, Findley $\sqcup$, et al. Lamotrigine monotherapy in newly diagnosed untreated epilepsy: a double-blind comparison with phenytoin. Epilepsia 1999;40:601-17.

30 Nieto-Barrera M, Brozmanova M, Capovilla G, et al. Lamictal vs Carbamazepine Study Group. A comparison of monotherapy with lamotrigine or carbamazepine in patients with newly diagnosed partial epilepsy. Epilepsy Res 2001;46:145-55.

31 Messenheimer J, Mullens EL, Giorgi L, et al. Safety review of adult clinical trial experience with lamotrigine. Drug Saf 1998;18:281-96.

32 Gilliam FG, Vazquez B, Sackellares JC, et al. An active-control trial of lamotrigine monotherapy for partial seizures. Neurology 1998;51:1018-25.

33 Biton V, Mirza W, Montouris G, et al. Weight change associated with valproate and lamotrigine monotherapy in patients with epilepsy. Neurology 2001;56:172-7.

34 Edwards KR, Sackellares JC, Vuong A, et al. Lamotrigine monotherapy improves depressive symptoms in epilepsy: a double-blind comparison with valproate. Epilepsy Behav $2001 ; 2: 28-36$.

35 Stephen LJ, Kwan P, Shapiro D, et al. Hormone profiles in young adults with epilepsy treated with sodium valproate or lamotrigine monotherapy. Epilepsia $2001 ; 42: 1002-6$

36 Barrett C, Richens A. Epilepsy and pregnancy: report of an epilepsy research foundation workshop. Epilepsy Res 2003:52:147-87.

37 Morrell MJ, Isojarvi J, Taylor AE, et al. Higher androgens and weight gain with valproate compared with lamotrigine for epilepsy. Epilepsy Res 2003;54:189-99.

38 Aldenkamp AP, Arends J, Bootsma HP, et al. Randomized double-blind parallel-group study comparing cognitive effects of a low-dose lamotrigine with valproate and placebo in healthy volunteers. Epilepsia 2002;43:19-26.

39 Aldenkamp AP, Krom MD, Reijs R. Newer antiepileptic drugs and cognitive issues. Epilepsia 2003:44(suppl 4):21-29.

40 Goldsmith DR, Wagstaff AJ, Ibbotson T, et al. Lamotrigine: a review of its use in bipolar disorder. Drugs 2003;63:2029-50.

41 Sabers A, Ohman I, Christensen J, et al. Oral contraceptives reduce lamotrigine plasma levels. Neurology 2003:61:570-71.

42 Schmidt D, Sachdeo R. Oxcarbazepine for treatment of partial epilepsy: a review and recommendations for clinical use. Epilepsy Behav 2000; 1:396-405

43 Bang L, Goa K. Oxcarbazepine: a review of its use in children with epilepsy Paediatr Drugs 2003;5:557-73.

44 Smith PEM. Clinical recommendations for oxcarbazepine. Seizure 2001;10:87-91.

45 Homberg V, Kowalik A, Schulze-Bonhage A. Ad-hoc Umstellung von Carbamazepin zu Oxcarbazepin. Effizienz und Verträglichkeit. Eine retropsektive Analyse. Nervenarzt 2001;72:918-23.

46 Sachdeo RC, Wasserstein A, Mesenbrink PJ, et al. Effects of oxcarbazepine on sodium concentration and water handling. Ann Neurol 2002:51:613-20.

47 Kutluay E, McCague K, D'Souza J, et al. Safety and tolerability of oxcarbazepine in elderly patients with epilepsy. Epilepsy Behav 2003;4:175-80.

48 Isoiärvi JI, Pakarinen AJ, Rautio A, et al. Serum sex hormone levels after replacing carbamazepine with oxcarbazepine. Eur J Clin Pharmacol 1995;47:461-4.
49 Bauer J, Blumenthal S, Reuber M, et al. Epilepsy syndrome, focus location, and treatment affect testicular function in men with epilepsy. Neurology 2004;62:243-6.

50 Shank RP, Gardocki JF, Streeter AJ, et al. An overview of the preclinical aspects of topiramate: pharmacology, pharmacokinetics, and mechanism of action. Epilepsia 2000;41:S3-S9.

51 Sachdeo RC, Reife RA, Lim P, et al. Topiramate monotherapy for partial onset seizures. Epilepsia 1997;38:294-300.

52 Rosenfeld WE, Sachdeo RC, Faught RE, et al. Long-term experience with topiramate as adjunctive therapy and as monotherapy in patients with partial onset seizures: retrospective survey of open-label treatment. Epilepsia 1997;38(suppl 1):S34-6.

53 Privitera MD, Brodie MJ, Mattson RH, et al. Topiramate, carbamazepine and valproate monotherapy: double-blind comparison in newly diagnosed epilepsy. Acta Neurol Scand 2003;107:165-75.

54 Gilliam FG, Veloso F, Bomhof MA, et al. A dose-comparison trial of topiramate as monotherapy in recently diagnosed partial epilepsy. Neurology 2003;60:196-202.

55 Waugh J, Goa KL. Topiramate: as monotherapy in newly diagnosed epilepsy. CNS Drugs 2003;17:985-92.

56 Cohen J. Levetiracetam monotherapy for primary generalized epilepsy Seizure 2003;12:150-3

57 Appleton RE, Peters AC, Mumford JP, et al. Randomised, placebo-controlled study of vigabatrin as first-line treatment of infantile spasms. Epilepsia 1999:40:1627-33

58 Kälviäinen R, Nousiainen I. Visual field defects with vigabatrin: epidemiology and therapeutic implications. CNS Drugs 2001;15:217-30

59 McDonagh J, Stephen U, Dolan FM, et al. Peripheral retinal dysfunction in patients taking vigabatrin. Neurology 2003;61:1690-4.

60 Jain KK. An assessment of zonisamide as an anti-epileptic drug. Expert Opin Pharmacother 2000; 1:1245-60.

61 Errante LD, Petroff OA. Acute effects of gabapentin and pregabalin on rat forebrain cellular GABA, glutamate, and glutamine concentrations. Seizure 2003;12:300-6.

62 Trinka E, Dilitz E, Unterberger I, et al. Non convulsive status epilepticus after replacement of valproate with lamotrigine. J Neurol 2002;249:1417-22.

63 Biraben A, Allain H, Scarabin JM, et al. Exacerbation of juvenile myoclonic epilepsy with lamotrigine. Neurology 2000;55:1758.

64 Carrazana EJ, Wheeler SD. Exacerbation of juvenile myoclonic epilepsy with lamotrigine. Neurology 2001;56:1424-5.

65 Rigoulot MA, Boehrer A, Nehlig A. Effects of topiramate in two models of genetically determined generalized epilepsy, the GAERS and the audiogenic wistar AS. Epilepsia 2003:44:14-19.

66 Cross JH. Topiramate monotherapy for childhood absence seizures: an open label pilot study. Seizure 2002;11:406-10.

67 Biton V, Montouris GD, Ritter F, et al. A randomized, placebo-controlled study of topiramate in primary generalized tonic-clonic seizures. Topiramate YTC Study Group. Neurology 1999;52:1330-7.

68 Wheless JW. Use of topiramate in childhood generalized seizure disorders. J Child Neurol 2000;15(suppl 1):S7-13

69 Abou-Khalil B, Hemdal P, Privitera MD. An open-label study of levetiracetam at individualised doses between 1000 and $3000 \mathrm{mg}$ day $(-1)$ in adult patients with refractory epilepsy. Seizure 2003;12:141-9.

70 Ben-Menachem E, Gilland E. Efficacy and tolerability of levetiracetam during 1-year follow-up in patients with refractory epilepsy. Seizure 2003;12:131-5.

71 Brunbech L, Sabers A. Effect of antiepileptic drugs on cognitive function in individuals with epilepsy. Drugs 2002;62:593-604.

72 Meador KJ. Newer anticonvulsants: dosing strategies and cognition in treating patients with mood disorders and epilepsy. J Clin Psychiatry 2003;64(suppl 8):30-4

73 Lee S, Sziklas V, Andermann F, et al. The effects of adjunctive topiramate on cognitive function in patients with epilepsy. Epilepsia 2003;44:339-47.

74 Mula M, Trimble MR, Lhatoo SD, et al. Topiramate and psychiatric adverse events in patients with epilepsy. Epilepsia 2003;44:659-63.

75 Aldenkamp AP, Baker G. A systematic review of the effects of lamotrigine on cognitive function and quality of life. Epilepsy Behav 2001;2:85-91.

76 Medeiros FA, Zhang XY, Bernd AS, et al. Angle-closure glaucoma associated with ciliary body detachment in patients using topiramate. Arch Ophthalmol 2003; 121:282-5

77 Bumb A, Diederich N, Beyenburg S. Adding topiramate to valproate therapy may cause reversible hepatic failure. Epileptic Disord 2003:5:157-9.

78 Cramer JA, De Rue K, Devinsky O, et al. A systematic review of the behavioral effects of levetiracetam in adults with epilepsy, cognitive disorders, or an anxiety disorder during clinical trials. Epilepsy Behav 2003;4:124-32.

79 Baver J, Oehring RD, Elger CE. Generalisiertes Exanthem bei Komedikation mit Levetiracetam. Z Epileptol 2001;14:23-4.

80 Nakken KO, Eriksson AS, Lossius R, et al. A paradoxical effect of levetiracetam may be seen in both children and adults with refractory epilepsy. Seizure 2003;12:42-6.

81 Kossoff EH, Bergey GK, Freeman JM, et al. Levetiracetam psychosis in children with epilepsy. Epilepsia 2001;42:1611-13.

82 Mula M, Trimble MR, Yuen A, et al. Psychiatric adverse events during levetiracetam therapy. Neurology 2003;61:704-6.

83 White JR, Walczak TS, Leppik IE, et al. Discontinuation of levetiracetam because of behavioral side effects: a case-control study. Neurology 2003;61:1218-21. 